\section{ORIGINAL RESEARCH}

R. Kadirvel

Y.H. Ding

D. Dai

D.A. Lewis

D.F. Kallmes

\title{
Intrinsic Pathway-Mediated Apoptosis in Elastase-Induced Aneurysms in Rabbits
}

\author{
BACKGROUND AND OBJECTIVES: The pathophysiology of saccular aneurysms is complex and multi- \\ factorial. The aim of the present study was to understand the mechanism of apoptosis in an \\ elastase-induced aneurysm model in rabbits.
}

\begin{abstract}
MATERIALS AND METHODS: Elastase-induced saccular aneurysms were created the origin of the right common carotid artery in 20 rabbits. Aneurysm samples were harvested at 2 and 12 weeks after creation. Expression of apoptosis-associated proteins, including caspases and bcl-2 proteins, were assessed by Western blot analysis ( $n=5$ at both time points). Terminal deoxynucleotidyltransferasemediated dUTP nick end-labeling (TUNEL) staining, which indicates the presence of apoptosis, was performed in tissue sections ( $n=5$ at both time points). The unoperated contralateral common carotid artery was used as a control.
\end{abstract}

RESULTS: Expression of active caspase-3, the final executioner of apoptosis, and caspase-9, the mediator of the intrinsic mitochondrial pathway, was observed in aneurysms at 2 weeks, whereas the expression of activated caspase-8, the mediator of the extrinsic death receptor pathway, was absent at both time points. Expression of antiapoptotic proteins, Bcl-2 and phospho-Bad, was down-regulated in aneurysms compared with controls at 2 weeks. None of these proteins were differentially expressed at 12 weeks. These results were confirmed by the presence of TUNEL-positive cells in some aneurysms at the early time point.

CONCLUSIONS: In this study of elastase-induced aneurysms in a rabbit model, activation of apoptosis is mediated predominantly by the $\mathrm{Bcl}-2$-mediated intrinsic pathway through the activation of caspase-9.

C erebral saccular aneurysms are characterized by profound structural and functional remodeling within the vessel wall, in some cases, associated with progressive arterial dilation and eventual rupture. Most vascular remodeling procedures, however, are adequate and prevent dilation and eventual rupture. ${ }^{1,2}$ Although the exact pathogenesis remains unknown, several environmental and genetic factors that may lead to continued irreversible destruction of the vessel wall have been recognized. ${ }^{3,4}$

Apoptosis is an energy-dependent form of cell death that occurs in a wide range of physiologic conditions, such as tissue remodeling, cell proliferation, and tumor regression. ${ }^{5-7}$ It is characterized by cytoplasmic and nuclear condensation, DNA fragmentation, membrane blebbing, and engulfment and lysosomal degradation of apoptotic bodies by phagocytosis. Apoptosis can be initiated by either the extrinsic pathway, intrinsic pathway, or both, leading to the activation of the final executioner, caspase- 3 , which results in fragmentation of the nucleus. ${ }^{8,9}$

We have developed an experimental elastase-induced aneurysm model in the rabbit carotid artery, which structurally mimics human intracranial saccular aneurysms. ${ }^{10-12}$ We previously reported the occurrence of apoptosis in the aneurysm sac after embolization with platinum coils. ${ }^{13,14}$ In the present

Received February 4, 2009; accepted after revision June 17.

From the Department of Radiology, Mayo Clinic, Rochester, Minnesota.

This study was supported in part by the funds from National Institutes of Health grant (2R01NS042646-04) and the American Heart Association postdoctoral fellowship (AHA0620016Z)

Please address correspondence to: David F. Kallmes, MD, Department of Radiology, Mayo Clinic College of Medicine, 200 First St SW, Rochester, MN 55905; e-mail: Kallmes. david@mayo.edu

Indicates open access to non-subscribers at www.ajnr.org

DOI 10.3174/ajnr.A1781 study, we aimed to evaluate the presence of apoptosis at different stages of aneurysm growth of the elastase-induced experimental aneurysm.

\section{Materials and Methods}

\section{Aneurysm Creation}

The Institutional Animal Care and Use Committee approved all procedures before initiation of the study. Elastase-induced saccular aneurysms were created in 20 New Zealand white rabbits (body weight, $3-4 \mathrm{~kg}$ ) by using the rabbit-elastase model. Detailed procedures for aneurysm creation have been described in depth elsewhere. ${ }^{15}$ Briefly, anesthesia was induced with an intramuscular injection of ketamine, xylazine, and acepromazine (75, 5, and $1 \mathrm{mg} / \mathrm{kg}$, respectively). Using a sterile technique, we exposed the right common carotid artery (RCCA) and ligated it distally. A 1- to 2-mm beveled arteriotomy was made, and a $5 \mathrm{~F}$ vascular sheath was advanced retrograde in the RCCA to a point approximately $3 \mathrm{~cm}$ cephalad to the origin of RCCA. A 3F Fogarty balloon was advanced through the sheath to the level of the origin of the RCCA with fluoroscopic guidance and was inflated with iodinated contrast material. Porcine elastase (Worthington Biochemical, Lakewood, New Jersey) was incubated within the lumen of the common carotid artery above the inflated balloon for 20 minutes, after which the catheter, balloon, and sheath were removed. The RCCA was ligated below the sheath entry site, and the incision was closed. Aneurysms were allowed to mature either for 2 weeks $(n=5$ each for molecular biology and terminal deoxynucleotidyltransferase [TdT] - mediated dUTP nick end-labeling [TUNEL] assay) or for 12 weeks ( $n=5$ each for molecular biology and TUNEL assay).

\section{Tissue Harvest}

Aneurysm samples were harvested at either 2 weeks $(n=10)$ or 12 weeks $(n=10)$ following aneurysm creation. Under general anesthesia, animals were euthanized by a lethal injection of sodium pento- 
barbital. The aneurysm and unoperated left common carotid artery (LCCA) were then harvested. The samples were placed in buffered formalin for TUNEL staining. If the aneurysms were relegated to molecular biology experiments, the aneurysm samples were dissected into 2 samples, including the neck and dome. These samples were kept frozen at $-70^{\circ} \mathrm{C}$.

\section{TUNEL Staining}

TUNEL staining was performed for specific labeling of nuclear DNA fragmentation, an important biochemical hallmark of apoptosis, with the DeadEnd Fluorometric TUNEL system (Promega Corp, Madison, Wisconsin). With this method, apoptosis can be quantified at the single-cell level; however, it does not detect the apoptotic cells at their early stages. The fixed vessels were dehydrated in an ascending series of ethanol, cleared in xylene, and embedded in paraffin. The paraffinembedded tissues were then sectioned at a $5-\mu \mathrm{m}$ thickness. The tissue sections were deparaffinized and rehydrated, followed by fixing them in $4 \%$ paraformaldehyde for 15 minutes. They were incubated with 20 $\mu \mathrm{g} / \mathrm{mL}$ of proteinase $\mathrm{K}$ for 20 minutes at room temperature, and then sections were rinsed with phosphate buffered solution (PBS) and kept in the equilibration buffer for 10 minutes at room temperature. They were incubated with $100 \mu \mathrm{L}$ of TdT reaction ( $\mathrm{rTdT}$ ) mix containing the rTdT enzyme and fluorescent-labeled nucleotides in a humid atmosphere for 1 hour at $37^{\circ} \mathrm{C}$. The reaction was terminated by immersing the slides in water for 15 minutes. The sections were then immersed in $2 \times$ SSC buffer for 15 minutes at room temperature to terminate the reaction and then were rinsed with PBS. The slides were analyzed by fluorescence confocal microscopy. Positive controls consisted of incubating sections with deoxyribonuclease I. A negative control was performed by incubating the section with $\mathrm{rTdT}$ reaction mix without the rTdT enzyme.

\section{Immunoblotting}

Frozen samples were pulverized under liquid nitrogen and extracted in an ice-cold lysis buffer (10-mmol/L sodium phosphate, $\mathrm{pH} 7.2$, 150-mmol/L sodium chloride, $1 \%$ Triton X-100, 0.1\% sodium dodecyl sulfate [SDS], $0.5 \%$ sodium deoxycholate, and $0.2 \%$ sodium azide with a protease inhibitor cocktail). After centrifugation at $10,000 \mathrm{~g}$ for 20 minutes at $4^{\circ} \mathrm{C}$, the protein concentration of the supernatant was determined (Pierce Biotechnology, Rockford, Illinois).

Total protein was fractionated on SDS polyacrylamide gel electrophoresis and transferred to a polyvinylidene difluoride membrane (Bio-Rad, Hercules, California). Membranes were incubated with monoclonal anti-bcl-2, phospho-Bad, caspase-8 (Cell Signaling Technology, Danvers, Massachusetts), rabbit polyclonal anticaspase-3, and caspase-9 (Cell Signaling Technology) overnight at $4^{\circ} \mathrm{C}$. The membranes were incubated with the appropriate secondary antibody; either a sheep anti-rabbit or goat anti-mouse immunoglobulin G (Bio-Rad) dilution conjugated to horseradish peroxidase. Immune complexes were visualized by using the enhanced chemiluminescence detection system (ECL Western Blotting Detection Reagents; GE Healthcare, Piscataway, New Jersey), and bands were measured by densitometric analysis of autoradiograph films (UNSCAN-IT software, Silk Scientific, Orem, Utah).

\section{Statistical Analysis}

Results are expressed as mean $\pm \mathrm{SD}$. The unoperated LCCAs were used as controls. Differences between groups were assessed by the Student $t$ test. Statistical significance was taken as a probability value

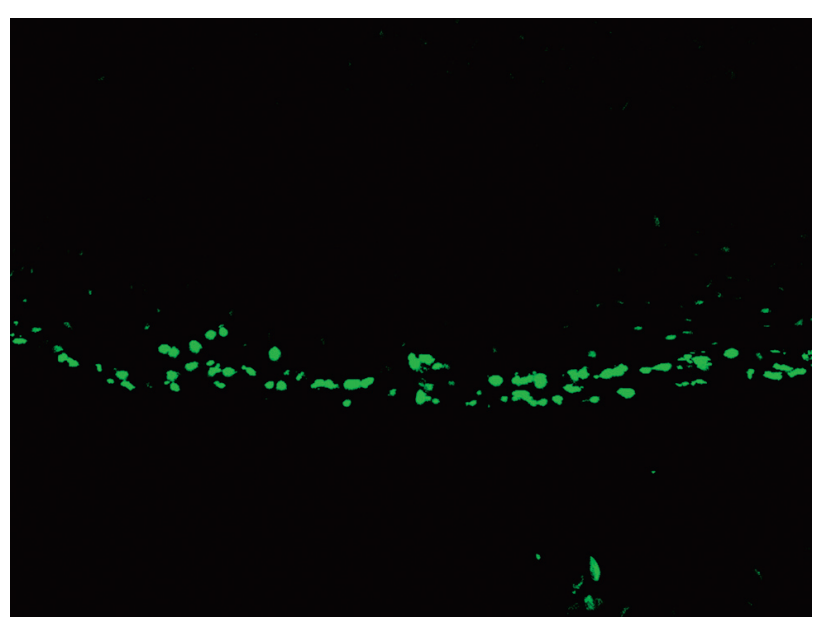

Fig 1. Terminal deoxynucleotidyltransferase-mediated dUTP nick end-labeling (TUNEL) staining. Immunofluorescence image of TUNEL staining at the distal neck of a 2-week aneurysm (original magnification $\times 150$ ).

of $<.05$. Each value was compared with the LCCA of the same time period, as a control. The values from the 2-week group and 12-week group were also compared.

\section{Results}

\section{TUNEL Staining}

The TUNEL assay was applied on tissue sections to detect apoptotic cells. At 2 weeks after aneurysm creation, 2 of 5 aneurysms $(40 \%)$ were positive for TUNEL staining (Fig 1). No TUNEL-positive cells were observed in any of the 12-week aneurysms.

\section{Expression of Caspases}

The expression of caspases was analyzed in control and aneurysm samples by using Western blotting. There was no difference in expression between aneurysms and controls for procaspase-3 (35 kDa), pro-caspase-8 (57 kDa and pro-caspase- 9 $(35 \mathrm{kDa})$ at either time point. Expression of cleaved activatedcaspase-3 $(17 \mathrm{kDa})$ and caspase- $9(17 \mathrm{kDa})$ was detected in the neck and dome of aneurysms compared with controls at 2 weeks but not at 12 weeks $(P<.001)($ Fig 2$)$.

\section{Expression of bcl-2 and Phospho-Bad}

Because the cleavage of pro-caspase- 9 to active cleaved caspase is associated with the expression of antiapoptotic proteins bcl-2 and phospho-Bad, we analyzed the expression of those proteins in the aneurysm samples. The levels of both bcl-2 and phospho-Bad were significantly lower in aneurysms at 2 weeks $(P<.001)$ compared with controls (Fig 3). At 12 weeks, the expressions of bcl-2 and phospho-Bad were not significantly different between aneurysms and controls.

\section{Discussion}

Development and growth of cerebral saccular aneurysms involves a complex remodeling of cells in the arterial wall. Apoptosis of vascular smooth muscle cells has been widely reported in the pathogenesis of atherosclerosis and abdominal aneurysms. ${ }^{16,17}$ Kondo et al, ${ }^{18}$ in a rat model of early cerebral aneurysm development, identified apoptotic medial smooth muscle cells, prompting those authors to posit an association 

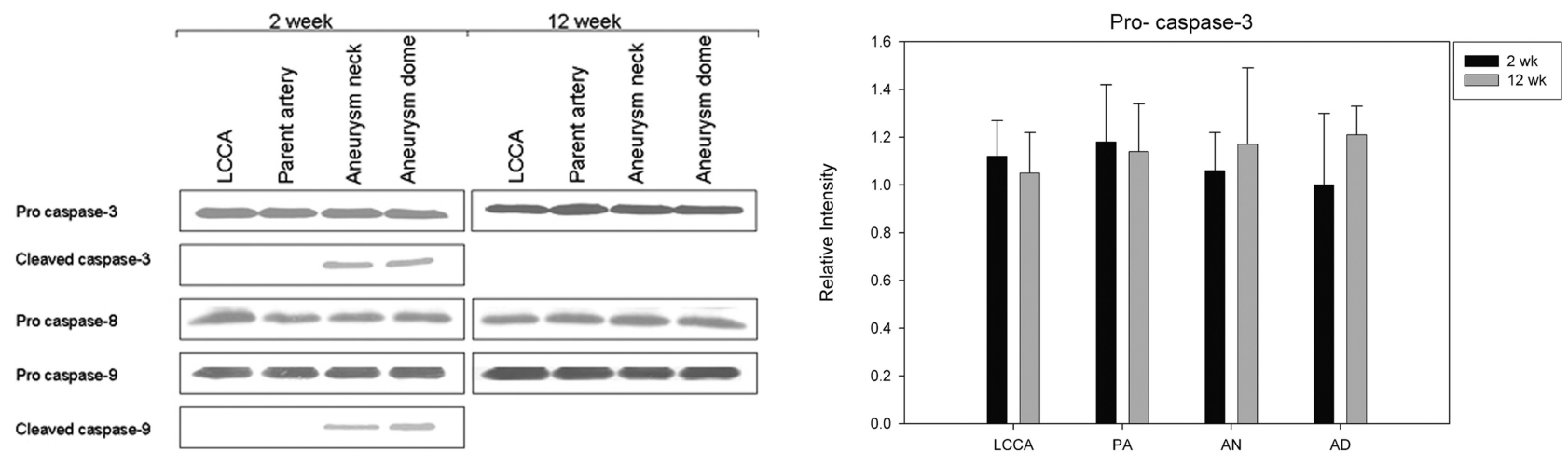

A

B
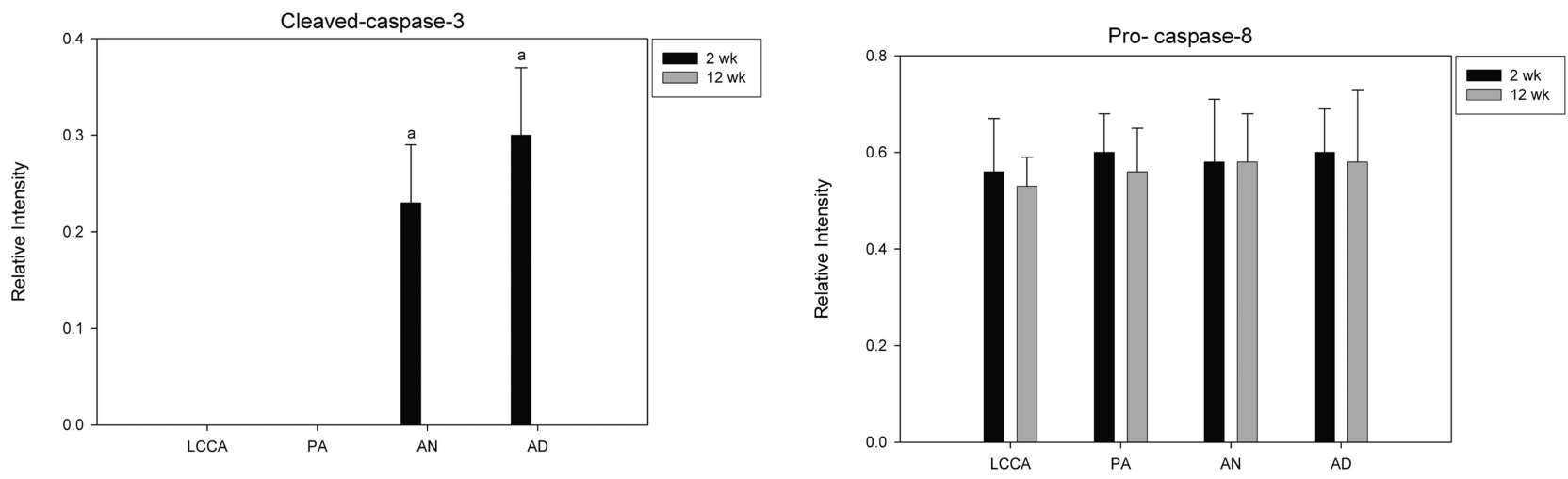

C

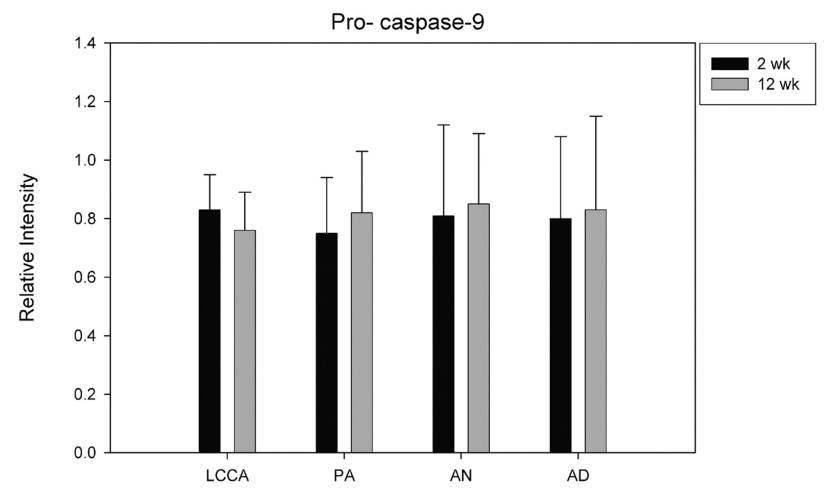

$\mathrm{E}$

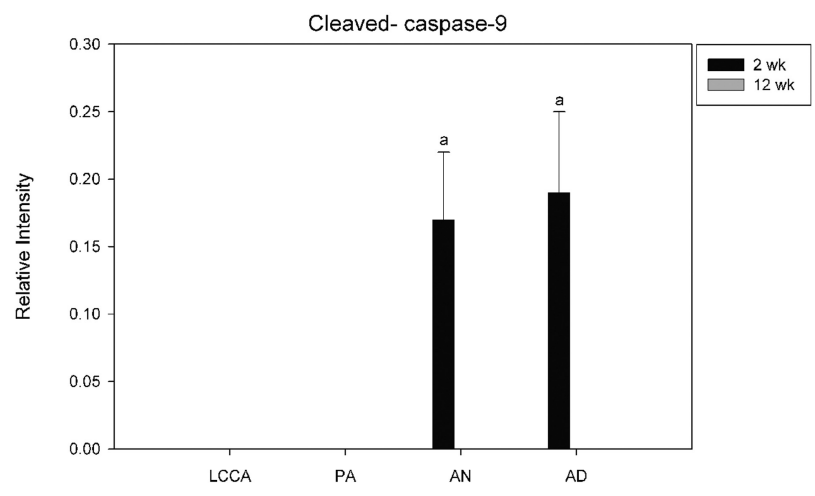

$\mathrm{F}$

Fig 2. Expression of proapoptotic proteins. $A$, Representative Western blot images of the expression of caspase family proteins from rabbits at 2 weeks and 12 weeks post-aneurysm creation. $B$, Densitometric analysis of the expression of procaspase-3 between control and experimental animals. $C$, Densitometric analysis of the expression of cleaved-caspase-3 between control and experimental animals. D, Densitometric analysis of the expression of procaspase-8 between control and experimental animals. E, Densitometric analysis of the expression of procaspase- 9 between control and experimental animals. F, Densitometric analysis of the expression of cleaved-caspase- 9 between control and experimental animals. LCCA indicates left common carotid artery; PA, parent artery; AN, aneurysm neck; $\mathrm{AD}$, aneurysm dome; a, $P<.05$ versus control; $\mathrm{b}, P<.05$ versus the corresponding 2-week group.

between apoptosis and aneurysm formation. ${ }^{19}$ The current study used a different animal model than the one in Kondo et al, in which saccular dilation of the proximal RCCA was achieved by distal ligation and proximal elastase injury. Thus, we studied the apoptotic activity of "formed" aneurysms to determine whether programmed cell death occurred after aneurysm formation.

Our results indicated an increase in apoptotic activity and a decrease in antiapoptotic activity early after aneurysm creation. Although not specifically studied in the current work, we have previously shown that our experimental aneurysms grow for approximately 3 weeks following creation. It is possible that ongoing aneurysm growth is promoted by apoptotic activity within the vessel wall, activity that diminishes with time and explains late stability of aneurysm size.

The mechanism that simulated apoptosis in our model remains unknown. Altered shear stress, which is markedly diminished in the aneurysm cavity, ${ }^{20}$ may lead to apoptosis of the aneurysm wall. ${ }^{21}$ Direct injury by the elastase may be responsible for the apoptosis. One might study this by adding a control group without elastase, but the vessels in that type of model simply involute. 


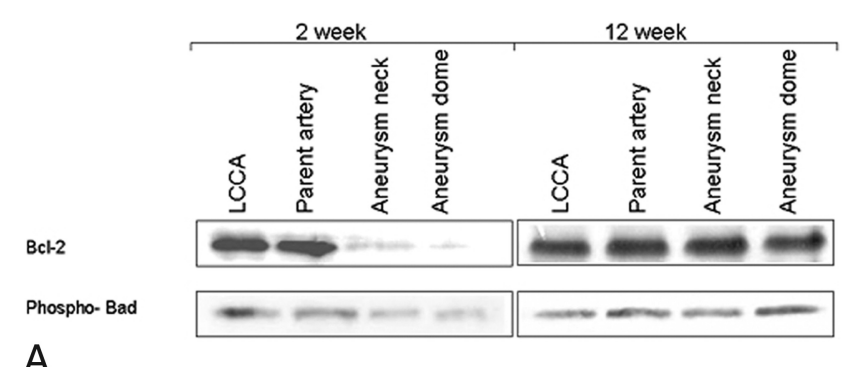

A

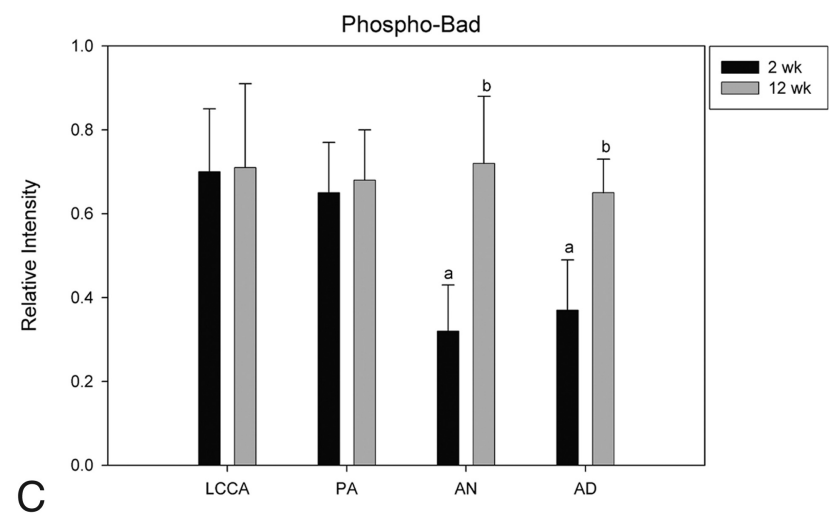

$\mathrm{Bcl}-2$

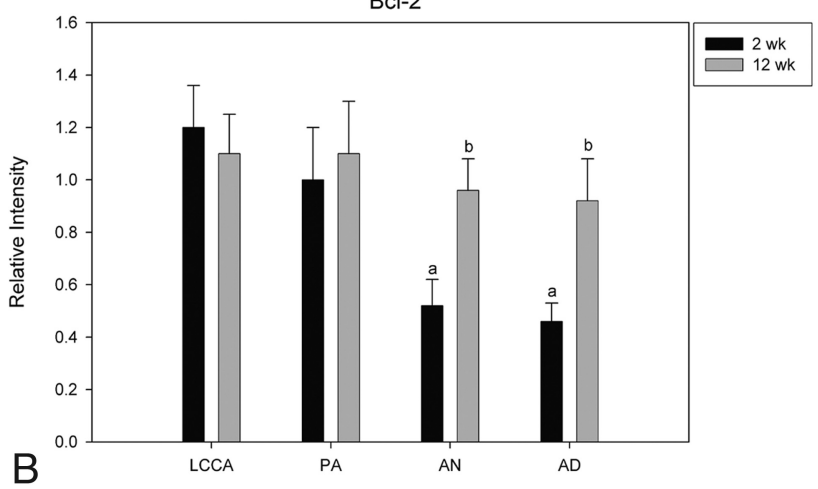

Fig 3. Expression of antiapoptotic proteins. $A$, Representative Western blot images of the expression of bcl-3 and phospho-Bad from rabbits at 2 weeks and 12 weeks post-aneurysm creation. $B$, Densitometric analysis of the expression of bcl-2 between control and experimental animals. $C$, Densitometric analysis of the expression of phospho-Bad between control and experimental animals.

Activation of caspases (cysteine aspartate-specific proteases), a family of cysteine proteinases, plays a central role in the process of apoptosis. The apoptotic caspases are classified as initiators or executioners, depending on their point of entry into the apoptotic cascade. The initiator caspases are the first to be activated in a particular death pathway, and they constitute the first step in a minimal 2-step cascade by activating the executioner caspases. Procaspases are synthesized as inactive zymogens, which are activated by cleavage.

Apoptosis can be initiated by either an extrinsic and/or intrinsic pathway, both leading to a final common pathway in which activated downstream caspases are involved in DNA fragmentation. The extrinsic pathway, known as the deathreceptor pathway, is mediated by the binding of ligands to receptors in the cell surface, which leads to the activation of caspase-8. In the mitochondrial intrinsic pathway, release of cytochrome $\mathrm{c}$ from the mitochondria to the cytosol forms an apoptotic complex, which in turn activates the apical caspase of the intrinsic pathway, caspase-9. Activation of caspase- 8 and/or caspase- 9 results in subsequent activation of the executioner caspase, caspase-3. This then leads to DNA fragmentation and death of the cell.

In the present study, cleaved-caspase-3 and cleavedcaspase- 9 were detected at the early time point, while cleavedcaspase- 8 was absent; this difference suggests that the apoptotic mechanism is mainly initiated by the intrinsic pathway. Both receptor- and mitochondrial-mediated apoptosis have been previously reported in intracranial aneurysms in humans. ${ }^{22,23}$ Up-regulation of the caspase-3 gene is closely im- plicated in the pathologic process of the ruptured intracranial aneurysm. ${ }^{23}$ Apoptosis in intracranial aneurysms is correlated with increased expression of phosphorylated c-Jun amino-terminal kinase and phosphorylated c-Jun. ${ }^{24}$

To further confirm the involvement of the mitochondrial pathway in the apoptosis of the elastase-induced aneurysm model, we measured the levels of antiapoptotic proteins, bcl-2 and phospho-Bad, which are associated with the intrinsic pathway. The levels of bcl-2 and phospho-Bad were found to be decreased in 2-week aneurysm samples only. A decreased level of bcl-2 protein lowers the mitochondrial membrane permeability, which leads to the release of cytochrome $\mathrm{c}$ to cytosol, the recruitment and activation of caspase- 9 .

The increased extent of the apoptotic mechanism soon after aneurysm creation indicates that apoptosis may play an important role in the progression of an aneurysm. Previous studies have shown that the endothelial cells along the wall of elastase-induced aneurysms were present at 2 weeks after creation and that the cells were dispersed at 12 weeks. ${ }^{20}$

\section{Limitations of the Study}

TUNEL-positive cells were identified in only 2 of 5 aneurysms after 2 weeks. TUNEL staining detects nuclear DNA strand breaks that happen at the final stages of apoptosis. TUNEL staining does not detect the initial stages of apoptosis. Another limitation is the plane of the histologic section, which does not contain all cells undergoing apoptosis. We analyzed the apoptosis at only 2 time points after creation. In the present study, 
the LCCA was used as the control, but we acknowledge that a sham-operated control might offer additional valuable data.

\section{References}

1. Bruno G, Todor R, Lewis I, et al. Vascular extracellular matrix remodeling in cerebral aneurysms. J Neurosurg 1998;89:431-40

2. Frosen J, Piippo A, Paetau A, et al. Remodeling of saccular cerebral artery aneurysm wall is associated with rupture: histological analysis of 24 unruptured and 42 ruptured cases. Stroke 2004;35:2287-93. Epub 2004 Aug 19

3. Inoue K, Mineharu Y, Inoue S, et al. Search on chromosome 17 centromere reveals TNFRSF13B as a susceptibility gene for intracranial aneurysm: a preliminary study. Circulation 2006;113:2002-10

4. Juvela S, Poussa K, Porras M. Factors affecting formation and growth of intracranial aneurysms: a long-term follow-up study. Stroke 2001;32:485-91

5. Selvendiran K, Tong L, Vishwanath S, et al. EF24 induces G2/M arrest and apoptosis in cisplatin-resistant human ovarian cancer cells by increasing PTEN expression. J Biol Chem 2007;282:28609-18. Epub 2007 Aug 7

6. Clarke MC, Figg N, Maguire JJ, et al. Apoptosis of vascular smooth muscle cells induces features of plaque vulnerability in atherosclerosis. Nat Med 2006;12:1075-80

7. Cho A, Mitchell L, Koopmans D, et al. Effects of changes in blood flow rate on cell death and cell proliferation in carotid arteries of immature rabbits. Circ Res 1997;81:328-37

8. Salvesen GS, Dixit VM. Caspases: intracellular signaling by proteolysis. Cell 1997;91:443-46

9. Bredesen DE, Rao RV, Mehlen P. Cell death in the nervous system. Nature 2006;443:796-802

10. Altes TA, Cloft HJ, Short JG, et al. 1999 ARRS Executive Council Award: creation of saccular aneurysms in the rabbit-a model suitable for testing endovascular devices. American Roentgen Ray Society. AJR Am J Roentgenol 2000;174:349-54

11. Kallmes DF, Fujiwara NH. New expandable hydrogel-platinum coil hybrid device for aneurysm embolization. AJNR Am J Neuroradiol 2002;23:1580-88

12. Short JG, Fujiwara NH, Marx WF, et al. Elastase-induced saccular aneurysms in rabbits: comparison of geometric features with those of human aneurysms. AJNR Am J Neuroradiol 2001;22:1833-37

13. Kadirvel R, Ding YH, Dai D, et al. Molecular indices of apoptosis activation in elastase-induced aneurysms after embolization with platinum coils. Stroke 2007;38:2787-94. Epub 2007 Aug 23

14. Dai D, Ding YH, Danielson MA, et al. Histopathologic and immunohistochemical comparison of human, rabbit, and swine aneurysms embolized with platinum coils. AJNR Am J Neuroradiol 2005;26:2560-68

15. Kallmes DF, Fujiwara NH, Yuen D, et al. A collagen-based coil for embolization of saccular aneurysms in a New Zealand white rabbit model. AJNR Am J Neuroradiol 2003;24:591-96

16. von Wnuck Lipinski K, Keul P, Lucke S, et al. Degraded collagen induces calpain-mediated apoptosis and destruction of the X-chromosome-linked inhibitor of apoptosis (xIAP) in human vascular smooth muscle cells. Cardiovasc Res 2006;69:697-705. Epub 2005 Oct 11

17. Liao S, Curci JA, Kelley BJ, et al. Accelerated replicative senescence of media smooth muscle cells derived from abdominal aortic aneurysms compared to the adjacent inferior mesenteric artery. J Surg Res 2000;92:85-95

18. Kondo S, Hashimoto N, Kikuchi H, et al. Apoptosis of medial smooth muscle cells in the development of saccular cerebral aneurysms in rats. Stroke 1998; 29:181-88, discussion 189

19. Stehbens WE. Apoptosis and matrix vesicles in the genesis of arterial aneurysms of cerebral arteries. Stroke 1998;29:1478-80

20. Kadirvel R, Ding YH, Dai D, et al. The influence of hemodynamic forces on biomarkers in the walls of elastase-induced aneurysms in rabbits. Neuroradiology 2007;49:1041-53

21. Qi YX, Qu MJ, Long DK, et al. Rho-GDP dissociation inhibitor alpha downregulated by low shear stress promotes vascular smooth muscle cell migration and apoptosis: a proteomic analysis. Cardiovasc Res 2008;80:114-22. Epub 2008 Jun 7

22. Jayaraman $\mathrm{T}$, Berenstein $\mathrm{V}, \mathrm{Li} \mathrm{X}$, et al. Tumor necrosis factor alpha is a key modulator of inflammation in cerebral aneurysms. Neurosurgery 2005;57:558-64

23. Guo $\mathrm{F}, \mathrm{Li} \mathrm{Z}$, Song $\mathrm{L}$, et al. Increased apoptosis and cysteinyl aspartate specific protease-3 gene expression in human intracranial aneurysm. J Clin Neurosci 2007;14:550-55

24. Takagi Y, Ishikawa M, Nozaki K, et al. Increased expression of phosphorylated c-Jun amino-terminal kinase and phosphorylated c-Jun in human cerebral aneurysms: role of the c-Jun amino-terminal kinase/c-Jun pathway in apoptosis of vascular walls. Neurosurgery 2002;51:997-1002, discussion 1002-04 